Online Supporting Information for

\title{
Quantized Thickening Process and Kinetics of Lamellar \\ Crystals of A Low Molecular Weight Poly(ethylene oxide)
}

Xiong-Feng Tang, Xiao-Jin Wen, Xue-Mei Zhai, Nan Xia, Wei Wang*,

The Key Laboratory of Functional Polymer Materials of Ministry of

Education and Institute of Polymer Chemistry, College of Chemistry,

Nankai University, Tianjin 300071, China

\author{
Gerhard Wegner \\ Max-Planck-Institute for Polymer Research, Ackermannweg 10, \\ Postfach 3148, D-55128, Mainz, Germany \\ Zhong-Hua $\mathrm{Wu}$
}

Beijing Synchrotron Radiation Facility, Institute of High Energy Physics,

Chinese Academy of Sciences, Beijing 100049, China

\section{Experimental section}

PEO samples. The low molecular weight poly(ethylene oxide) was purchased from Fluka and used without further purification. Its absolute molecular weight $\left(\bar{M}_{n}=5000\right)$ and the polydispersity index $\left(\bar{M}_{w} / \bar{M}_{n}=1.008\right)$ were precisely determined using matrix-assisted laser desorption ionization time-of-flight (MALDI-TOF) mass spectrometer (Bruker Reflex II-TOF). The PEO possess one methoxy and one hydroxyl end groups. The extended chain length of PEO is $L=l_{\mathrm{m}} N=31.6 \mathrm{~nm}$ and the degree of polymerization is $N=114 .{ }^{1}$ The folded lengths of integer 
folded lamellae can be calculated by $l(\mathrm{n})=L /(\mathrm{n}+1)$, where $\mathrm{n}$ is the number of folds (quantized folding).

Small Angle X-ray Scattering (SAXS). SAXS experiments were performed on a SAXS system in Max-Planck-Institute for Polymer Research. The system is composed of a two-dimensional detector (Brucker Histar), a mirror (Osmic) and a rotating anode X-ray generator (Rigaku Mikro max 007) operated at $30 \mathrm{kV}$ and $10 \mathrm{~mA}$. The recording time for each measurement was $1800 \mathrm{~s}$. The wavelength of the incident $\mathrm{X}$-ray beam from $\mathrm{Cu} \mathrm{K} \alpha$ radiation is $\lambda=0.154 \mathrm{~nm}$. The distance between the sample and the detector was $1800 \mathrm{~mm}$. The long period was determined from the position of peak maxima in Lorentz-corrected intensity curves $\left(I q^{2}\right.$ vs $q$, where $q=4 \pi \sin \theta / \lambda$ and $2 \theta$ is the scattering angle).

\section{Sample preparation and measurement conditions for SASX} measurements. The PEO samples for SAXS were prepared in a differential scanning calorimeter (Perkin-Elmer Diamond). The samples were sealed in a standard aluminum pan. The sealed samples were at first heated to $75^{\circ} \mathrm{C}$ for $10 \mathrm{~min}$ to erase thermal history, and then quenched to $10{ }^{\circ} \mathrm{C}$ at cooling rate of $100{ }^{\circ} \mathrm{C} / \mathrm{min}$ and crystallized for 2 hours at $10{ }^{\circ} \mathrm{C}$. The treated samples were transferred special sample holders for the further measurements. In the further measurements the following conditions were used: 1 . Heat in a stepwise manner the samples from room temperature to 
the melting point. The measurements were performed at each temperature to monitor the changes of morphology with temperature. 2. Rapidly heat the samples to the test temperature and measure the changes of morphology with time at the constant temperature.

(1) H. Tadokoro, Y. Chatani, T. Yoshiharo, S. Tamara,S. Nurahashi, Makromol. Chem. 1964, 73, 109.

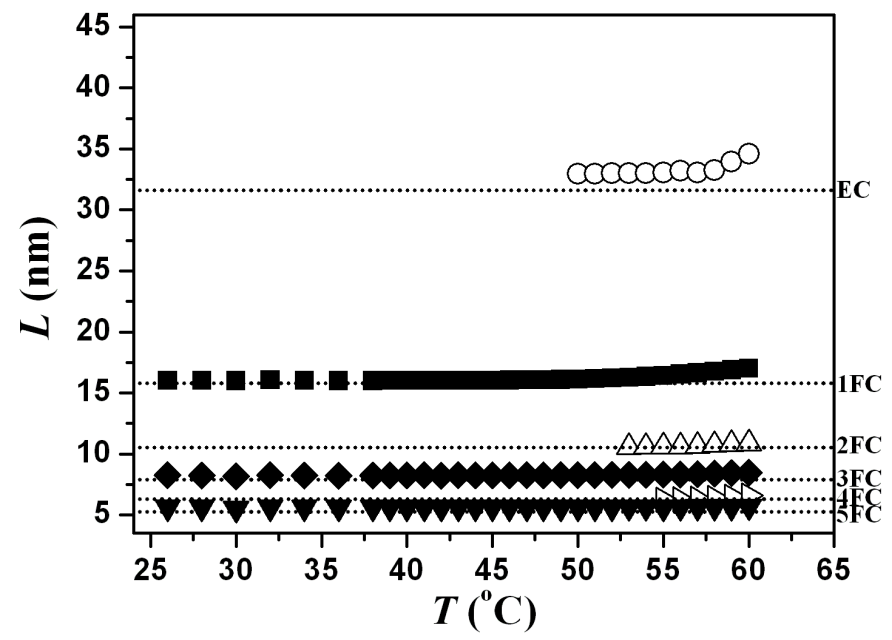

Figure S1. Long periods of as a function of temperature.

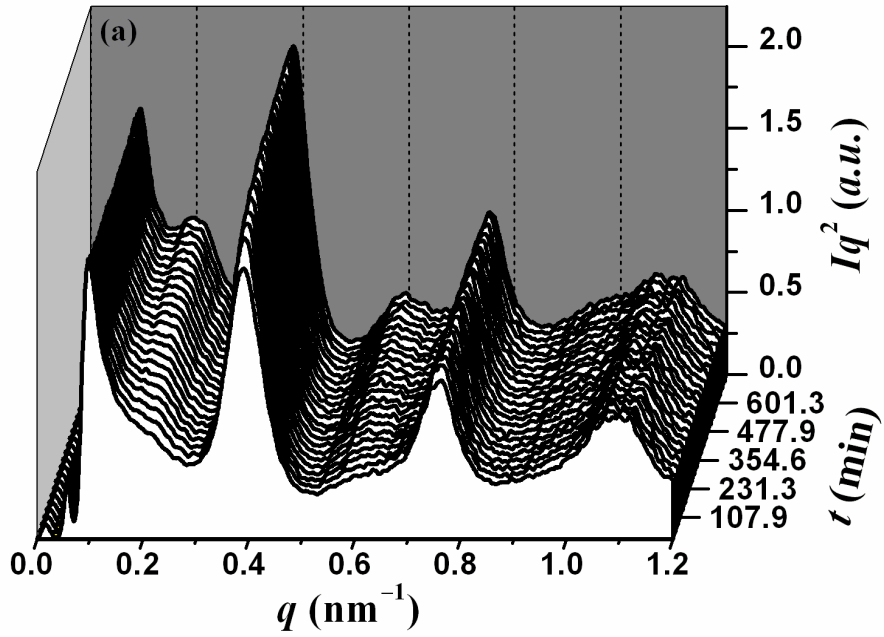



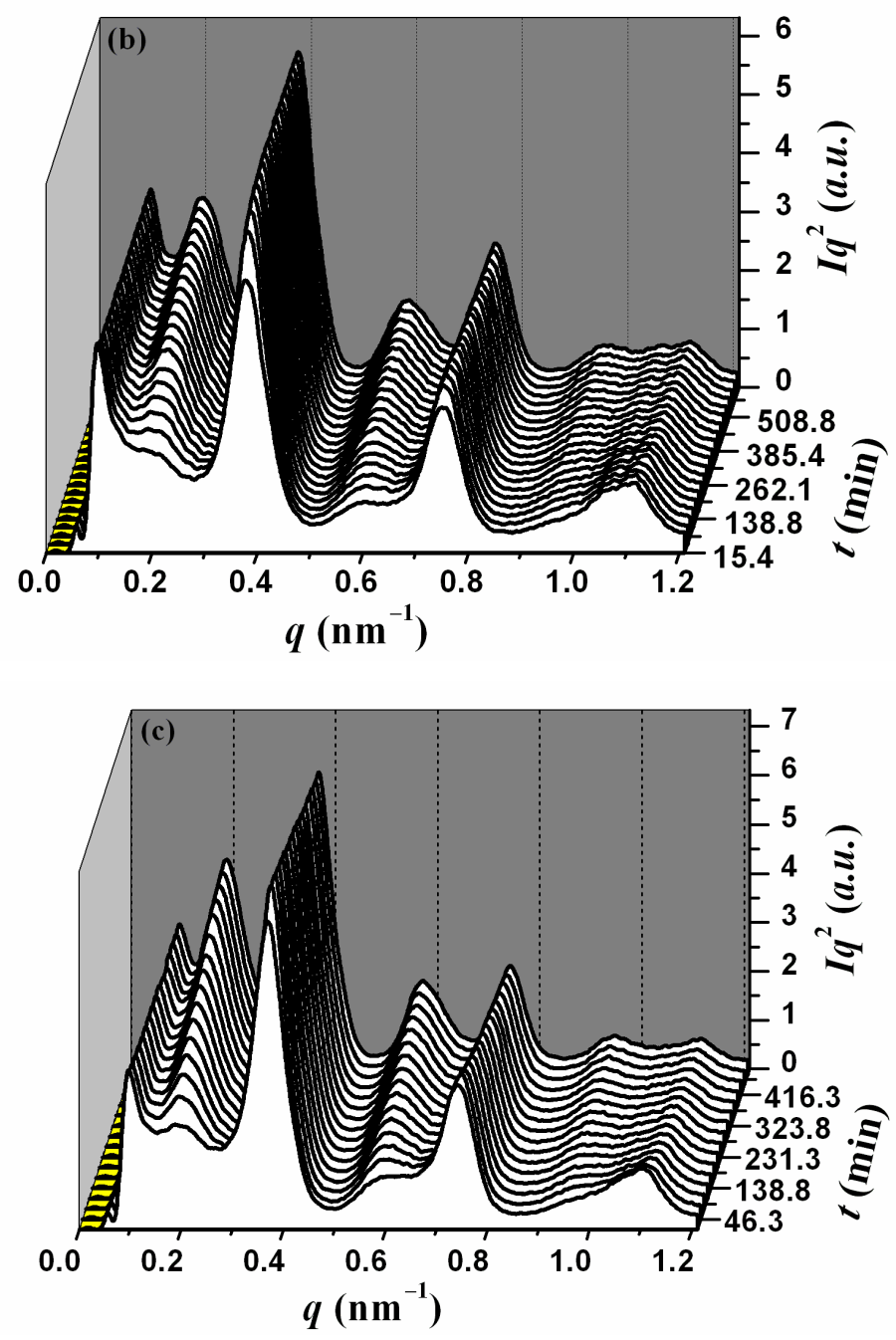

Figure S2, Time evolution of Lorentz-corrected SAXS intensity profiles for the samples annealed at $54{ }^{\circ} \mathrm{C}(\mathrm{a}), 57^{\circ} \mathrm{C}$ (b) and $58^{\circ} \mathrm{C}$ (c). 


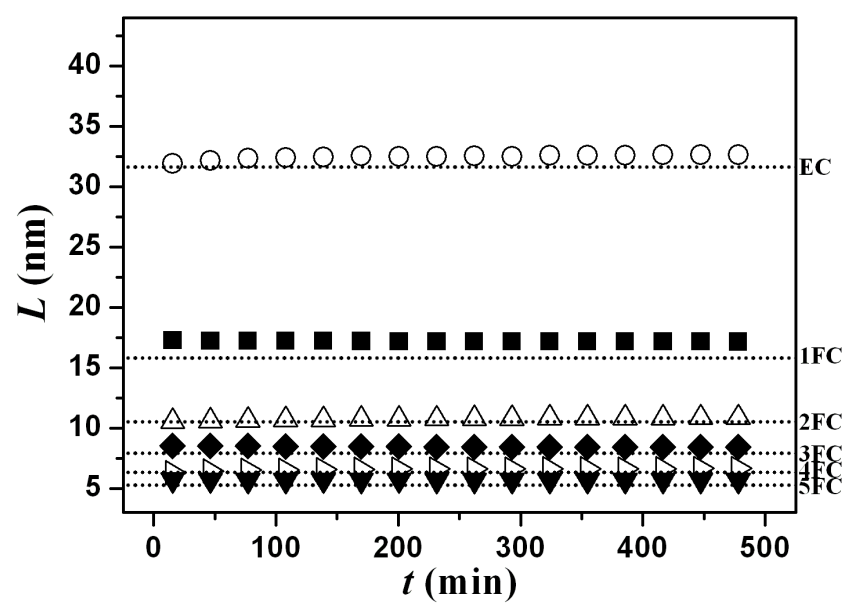

Figure S3. Changes in long period with the annealing time for PEO samples annealed at $58{ }^{\circ} \mathrm{C}$

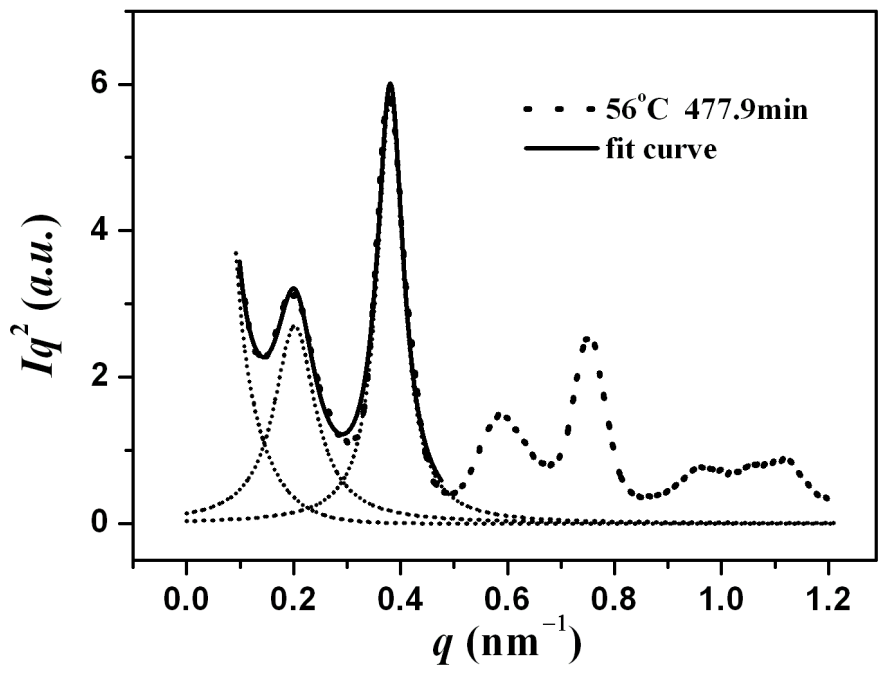

Figure S4. Nonlinear fit to a Lorentz-corrected SAXS intensity profile for the sample annealed at $56{ }^{\circ} \mathrm{C}$. 